\title{
Vasculitis cutánea de vasos pequeños. Revisión clínica en 32 casos
}

\author{
Donaldo López de Maturana L, Patricio Amaro B, \\ Laura Segovia G, Claudia Balestrini D. \\ Clinical features of 32 patients \\ with cutaneous small vessel vasculitis
}

Background: The skin is a common target of small vessel vasculitis, with a wide assortment of pathological changes. This condition is usually associated to systemic diseases. Aim: To report the clinical and pathological features of patients with cutaneous small vessel vasculitis. Material and methods: A retrospective review of 32 patients with a pathological diagnosis of cutaneous vasculitis. Results: Seventy two percent of patients were women. Cutaneous lesions were mainly located in the lower limbs (94\%). The most common lesion was palpable purpura (62\%). Connective tissue diseases and systemic vasculitis were the most commonly associated systemic diseases. Conclusions: Palpable purpura is the most common manifestation of cutaneous small vessel vasculitis, that is usually associated to connective tissue diseases or systemic vasculitis (Rev Méd Chile 2004; 132: 165-70).

(Key Words: Connective tissue diseases; Purpura; Vasculitis)

Recibido el 16 de abril, 2003. Aceptado en versión corregida el 24 de noviembre, 2003. Servicio de Dermatología y Anatomía Patológica. Hospital Barros Luco.

L a vasculitis cutánea de vasos pequeños (VCVP) corresponde a una inflamación vascular que afecta, habitualmente, a vénulas postcapilares como consecuencia de un proceso que suele estar mediado por complejos inmunes ${ }^{1}$. Histopatológicamente se caracteriza por inflamación segmentaria angiocéntrica, tumefacción celular endotelial, leucocitoclasia y necrosis fibrinoide de la pared vascular (Figura 1$)^{2}$, lo cual se traduce en la piel con manifestaciones diversas, siendo la más común el púrpura palpable. La VCVP puede estar circunscrita a la piel, pero en

Correspondencia a: Dr. Donaldo López de Maturana. Las Urbinas 81 Depto. 2- A, Providencia. Santiago. Fax: 2338304. E mail: donaldo@dermamed.cl muchos casos puede tener expresión sistémica de magnitud variable ${ }^{3,4}$.

El objetivo del presente trabajo es mostrar las características clínico patológicas de 32 pacientes con VCVP, haciendo énfasis en su relación a enfermedades sistémicas.

\section{Material y MÉTodo}

Se seleccionaron 32 casos de VCVP vistos en el Servicio de Dermatología del Hospital Barros Luco, entre los años 1988-1996, sobre la base de los siguientes criterios histopatológicos (en muestras teñidas con hematoxilina eosina).

a) Tumefacción de células endoteliales de vasos pequeños en dermis. 


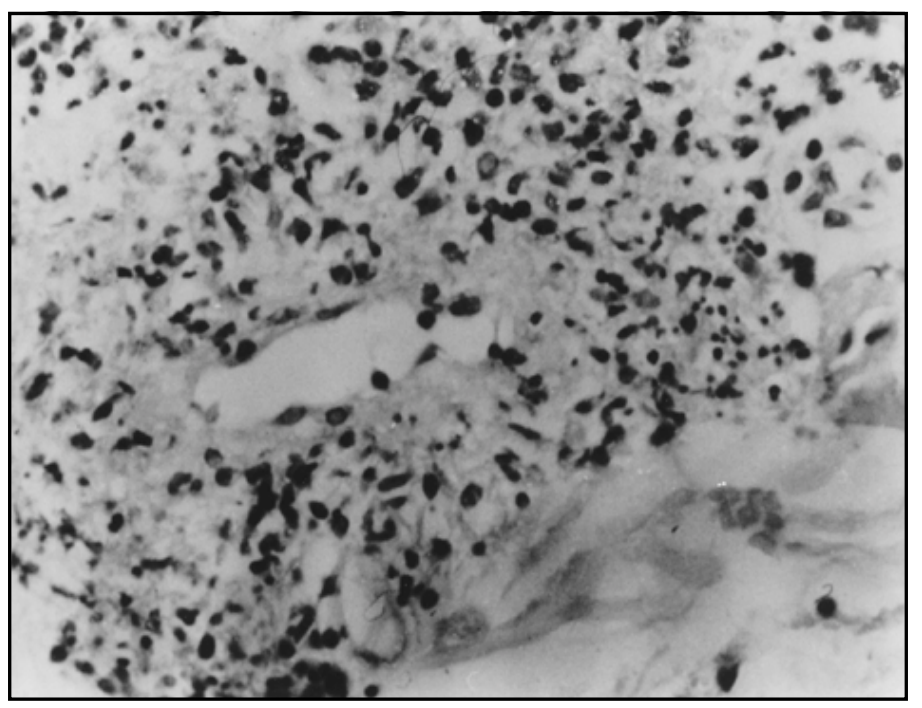

Figura 1. Vasculitis cutánea. Tumefacción endotelial, infiltrado inflamatorio mixto y polvo nuclear (aumento 40 X).

b) Infiltrado vascular y perivascular predominantemente neutrofílico.

c) Fragmentación nuclear de neutrófilos (leucocitoclasia).

En todos los casos se revisaron las fichas médicas, registrando las manifestaciones clínicas y la asociación a eventos desencadenantes o enfermedades sistémicas, también se revisaron las placas histológicas en búsqueda de hallazgos histopatoló- gicos de importancia como necrosis fibrinoide, extravasación de glóbulos rojos y trombosis.

\section{RESULTADOS}

Veintitrés pacientes eran mujeres (71,8\%). La edad promedio fue 43,5 años (fluctuó entre 18 y 80 años). Las lesiones cutáneas más frecuentes fueron: púrpura palpable (62\%) (Figura 2), mácula

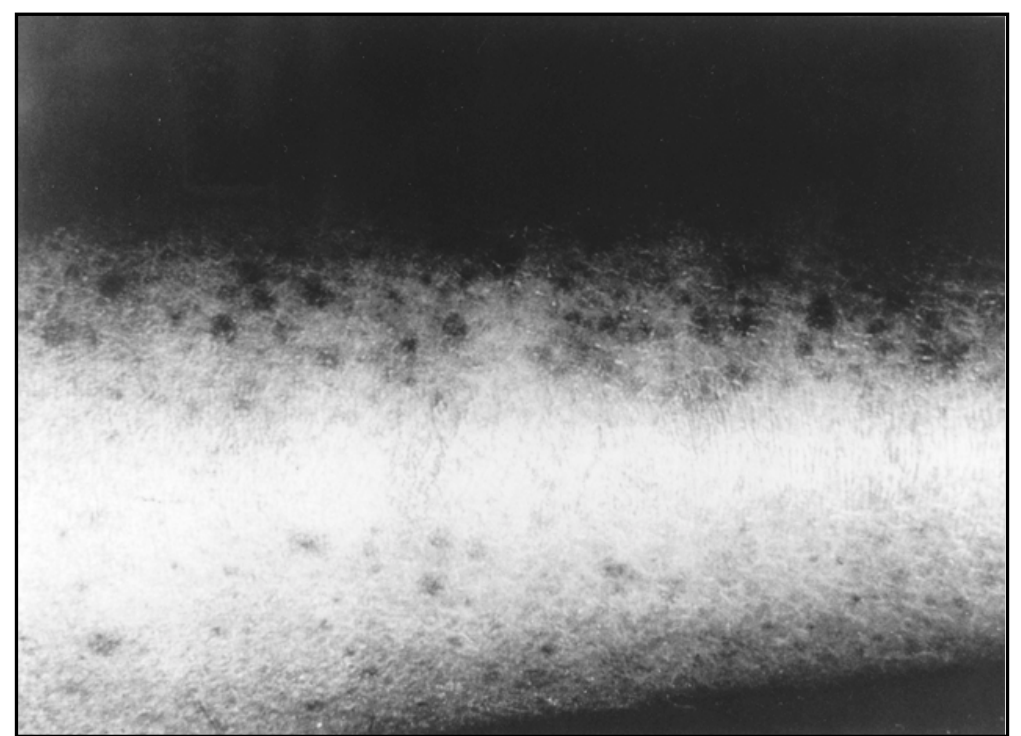

Figura 2. Púrpura palpable en extremidades inferiores. 
eritematosa $(34,3 \%)$ y ronchas $(15,6 \%)$ (Tabla 1$)$ y su ubicación más habitual fue en extremidades inferiores $(93,7 \%)$, tronco $(18,7 \%)$ y generalizadas $(18,7 \%)$.

En 20 casos (62,5\%) se identificó un agente etiológico involucrado (Tabla 2): medicamentos en 3 casos (9,4\%); infecciones del tracto respiratorio en 1 caso (3,1\%); enfermedades sistémicas: artritis reumatoide (AR) en 3 casos $(9,4 \%)$, lupus eritematoso sistémico (LES) (1 caso, 3,1\%), esclerodermia sistémica (1 caso, 3,1\%), vasculitis sistémica (5 casos, 15,6\%) y misceláneas (6 casos, 18,8\%).

Entre los exámenes de laboratorio, los resultados más significativos fueron una velocidad de sedimentación globular elevada en 18 casos

\section{Tabla 1. Tipo de lesión cutánea en vasculitis cutánea de vaso pequeño (32 casos )*}

\begin{tabular}{|lcc|}
\hline Tipo de lesión & № casos & $\begin{array}{c}\text { Porcentaje del } \\
\text { total de casos }\end{array}$ \\
\hline Púrpura palpable & 20 & 62,5 \\
Mácula eritematosa & 11 & 34,3 \\
Roncha & 5 & 15,6 \\
Ulcera & 5 & 15,6 \\
Petequia & 3 & 9,3 \\
Otras (vesículas, pústulas, & 4 & 12,5 \\
livedo reticular) & & \\
\hline
\end{tabular}

*En un número importante de pacientes coincidió más de un tipo de lesión.

Tabla 2. Eventos desencadenantes y enfermedades asociadas a vasculitis cutánea de vaso pequeño (32 casos)

\begin{tabular}{|c|c|c|c|}
\hline $\begin{array}{l}\text { Evento desencadenante o } \\
\text { enfermedad asociada }\end{array}$ & $\begin{array}{l}\text { № } \\
\text { casos }\end{array}$ & $\begin{array}{l}\text { Porcentaje del } \\
\text { total de casos }\end{array}$ & \\
\hline $\begin{array}{l}\text { Idiopática } \\
\text { Enfermedades del tejido } \\
\text { conectivo }\end{array}$ & $\begin{array}{r}12 \\
5\end{array}$ & $\begin{array}{l}37,5 \\
15,6\end{array}$ & $\begin{array}{l}\text { AR (3) } \\
\text { LES (1) } \\
\text { Esclerodermia (1) }\end{array}$ \\
\hline Vasculitis primarias & 5 & 15,6 & $\begin{array}{l}\text { Schönlein Henoch (2) } \\
\text { Churg Strauss (1) } \\
\text { G Wegener (1) } \\
\text { P Microscópica (1) }\end{array}$ \\
\hline Drogas & 3 & 9,4 & $\begin{array}{l}\text { Amoxicilina, paracetamol (1) } \\
\text { Penicilina sódica (1) } \\
\text { Dipirona, diclofenaco (1) }\end{array}$ \\
\hline Infecciones & 1 & 3,1 & $\begin{array}{l}\text { Infección del tracto } \\
\text { respiratorio superior }\end{array}$ \\
\hline Otras & 6 & 18,8 & $\begin{array}{l}\text { Síndrome hipereosinofílico (1) } \\
\text { Púrpura hiperglobulinémico (1) } \\
\text { Pioderma gangrenoso (1) } \\
\text { Colitis pseudomembranosa (1) } \\
\text { Enfermedad de Behçet (1) } \\
\text { Mieloma múltiple (1) }\end{array}$ \\
\hline
\end{tabular}


(56,2\%), C3 disminuido en 7 casos $(21,9 \%)$ y C4 en 4 casos $(12,5 \%)$ (asociados principalmente a urticaria vasculítica y al púrpura hiperglobulinémico). El factor reumatoide (FR) fue positivo en 3 pacientes $(9,3 \%)$ que correspondieron a los casos de artritis reumatoide (AR). El estudio de ANCA se realizó en 10 pacientes y resultó positivo en 1 caso de granulomatosis de Wegener (c-ANCA).

$\mathrm{Al}$ revisar las placas histopatológicas se observó: extravasación de glóbulos rojos en 27 casos (84,3\%), necrosis fibrinoide en 13 casos $(40,6 \%)$ y trombosis en 5 casos (15,6\%). En la Tabla 3 se comparan los casos con y sin necrosis fibrinoide, mostrando los eventos desencadenantes y enfermedades sistémicas asociadas en cada grupo.

\section{DisCUSIÓN}

La VCVP puede expresarse con lesiones diversas, pero el púrpura palpable es la más frecuente y así ocurrió también en nuestra casuística. Tanto el púrpura palpable como las úlceras se han relacionado a vasculitis cutáneas de curso agudo, a diferencia de las ronchas que suelen ser un marcador clínico de cronicidad y a asociarse a enfermedades autoinmunes, en especial cuando cursan con hipocomplementemia ${ }^{5,6}$.

Los factores etiológicos asociados a VCVP encontrados en nuestro trabajo, comprendieron medicamentos, infecciones y enfermedades sistémicas crónicas. La frecuente automedicación, la polifarmacia y el uso de fármacos en enfermedades, que por sí solas pueden causar VCVP, muchas veces impiden definir con certeza el agente involucrado. De acuerdo con la mayoría de los estudios, observamos la presencia significativa de enfermedades del tejido conectivo (AR, LES y

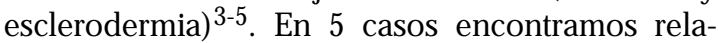
ción con vasculitis sistémicas primarias 7,8 , que revisten gran importancia por su gravedad y de su diagnóstico precoz depende, en gran medida, el pronóstico del paciente; en ellas ha sido de gran utilidad el estudio de los ANCA, que suelen ser marcadores serológicos de vasculitis sistémicas primarias $^{9}$. A pesar que no fue realizado en todos nuestros pacientes, encontramos c-ANCA positivo en un paciente con granulomatosis de Wegener, quien presentó lesiones purpúricas y úlceras en ambas piernas, cuya actividad fue paralela al compromiso multisistémico. Si bien en la granulomatosis de Wegener el marcador histológico clási-

Tabla 3. Eventos desencadenantes y enfermedades asociadas en pacientes con VCVP. Análisis comparativo de pacientes con y sin Necrosis Fibrinoide (N F) en el estudio histopatológico

\begin{tabular}{|lll|}
\hline $\begin{array}{l}\text { Eventos } \\
\text { desencadenantes y } \\
\text { enfermedades asociadas }\end{array}$ & $\begin{array}{l}\text { Con NF } \\
\mathrm{n}=13\end{array}$ & $\begin{array}{l}\text { Sin NF } \\
\mathrm{n}=19\end{array}$ \\
\hline Idiopáticas & & \\
Drogas & 3 & 9 \\
Infecciones & 2 & 1 \\
Vasculitis sistémicas & 1 & - \\
AR & 3 & 2 \\
LES & 2 & 1 \\
Esclerodermia & - & 1 \\
Misceláneas & - & 1 \\
& 2 & 4 \\
& (Behçet, síndrome & (Colitis \\
& hipereosinofílico) & pseudomembranosa, \\
& & Pioderma gangrenoso, \\
& & Púrpura hiperglobulinémico, \\
\hline
\end{tabular}


co y diagnóstico es una inflamación granulomatosa en el parénquima pulmonar comprometido, en la piel la manifestación más común es una VCVP con ausencia de granulomas, especialmente en lesiones cutáneas de tipo purpúrico ${ }^{10,11}$. Identificamos también un grupo de enfermedades misceláneas (enfermedad de Behçet, pioderma gangrenoso, síndrome de hipereosinofílico y púrpura hiperglobulinémico), que si bien son asociaciones menos frecuentes, presentan un claro componente autoinmune y su relación con la VCVP ha sido discutida por algunos autores ${ }^{12-14}$. La enfermedad de Behçet y el pioderma gangrenoso son entidades poco comunes, que se expresan en la piel con elementos ulcerativos y pustulosos, que suelen mostrar una reacción vascular neutrofílica prominente en la histopatología, pero en ocasiones, como en nuestros casos, puede observarse daño vascular más significativo $2,12,13$. Aunque no fue observado en nuestra serie, las neoplasias (linfomas, leucemias, etc) ${ }^{15}$ también deben considerarse ante un paciente con VCVP.

Basados en criterios histopatológicos ampliamente aceptados ${ }^{16}$ (infiltrado neutrofílico vascular y perivascular, polvo nuclear, necrosis fibrinoide, tumefacción endotelial, trombosis y extravasación de glóbulos rojos), las diversas series clínicas sobre VCVP muestran una asociación importante a enfermedades sistémicas (Tabla 4) ${ }^{4,5,17,18}$. Sin embargo, existe poca información referente a la correlación del daño cutáneo con el compromiso de otros órganos. Al comparar nuestra serie con la de Sánchez ${ }^{4}$ (ambas realizadas en centros hospitalarios), no se aprecian diferencias en cuanto a asociación a enfermedades sistémicas, a pesar que no exigimos la presencia de necrosis fibrinoide como criterio de inclusión al grupo en estudio (sólo $40,6 \%$ de los pacientes la presentó versus $100 \%$ en la serie de Sánchez). Por otro lado, tampoco observamos diferencias significativas, en este sentido, al comparar nuestros pacientes con y sin necrosis fibrinoide. Respecto de este mismo tema, Cribier y cols ${ }^{19}$, en un estudio de 184 pacientes con vasculitis leucocitoclástica concluyeron que la intensidad de la necrosis vascular y la profundidad de la vasculitis no se correlacionaron con un mayor grado de compromiso sistémico, mientras que Sais $^{20}$, en su trabajo sobre factores pronósticos en 160 pacientes con vasculitis leucocitoclástica, no encontró una asociación significativa entre las variables histológicas y el pronóstico (los resultados de este estudio identificaron fiebre, parestesia y ausencia de lesiones dolorosas como factores de riesgo de compromiso sistémico). Todos estos argumentos sugieren que la severidad del daño vascular cutáneo en la VCVP no es predictivo de mayor compromiso sistémico y que la variabilidad de los hallazgos histopatológicos depende más de la evolución del proceso inflamatorio cutáneo a través del tiempo, que del evento desencadenante o condición sistémica asociada.

Tabla 4. Condiciones asociadas a VCVP. Tabla comparativa con diversas series clínicas

\begin{tabular}{|lccccc|}
\hline & $\begin{array}{c}\text { Winkelmann } \\
(1964) \\
\mathrm{n}=38\end{array}$ & $\begin{array}{c}\text { Handel }^{16} \\
(1975) \\
\mathrm{n}=31\end{array}$ & $\begin{array}{c}\text { Callen }^{5} \\
(1984) \\
\mathrm{n}=82\end{array}$ & $\begin{array}{c}\text { Sánchez }^{4} \\
(1995) \\
\mathrm{n}=101\end{array}$ & $\begin{array}{c}\text { Presente } \\
\text { estudio } \\
\mathrm{n}=32\end{array}$ \\
\hline Idiopáticas & $39 \%$ & $61 \%$ & $54 \%$ & $26 \%$ & $37,5 \%$ \\
Drogas & $59 \%$ & $16 \%$ & $10 \%$ & $13 \%$ & $9,4 \%$ \\
Infecciones & 0 & 0 & $10 \%$ & $9 \%$ & $3,1 \%$ \\
Infecciones vs droga & 0 & 0 & $3 \%$ & 0 & 0 \\
Enfermedades del tejido conectivo & 0 & $10 \%$ & $21 \%$ & $21 \%$ & $15,6 \%$ \\
Neoplasias & 0 & $6,5 \%$ & 0 & $8 \%$ & 0 \\
Vasculitis sistémicas primarias & 0 & 0 & 0 & $16 \%$ & $15,6 \%$ \\
Otras & $2 \%$ & $6,5 \%$ & $2 \%$ & $8 \%$ & $18,8 \%$ \\
\hline
\end{tabular}


En resumen, la VCVP puede ser reflejo de una gran variedad de condiciones, algunas de importante repercusión sistémica, situación que debe considerarse en su manejo. Su diagnóstico debe

\section{REFERENCIAS}

1. Lotti T, Ghersetich I, Comacchi C, Jorizzo J. Cutaneous small - vessel vasculitis. J Am Acad Dermatol 1998; 39: 667-87.

2. Jorizzo J, Solomon AR, Zanow MD, Leshin B. Neutrophilic vascular reactions. J Am Acad Dermatol 1988; 19: 983-1005.

3. CALLEN JP, Ekenstam E. Cutaneous leukocitoclastic vasculitis: Clinical experience in 44 patients. Southern Med J 1987; 80: 848-51.

4. Sánchez N, Von Hale H, Su WP. Clinical and histopathologic spectrum of necrotizing vasculitis. Report of findings in 101 cases. Arch Dermatol 1985; 121: 220-4.

5. Ekenstam E, CALLen JP. Cutaneous leukocytoclastic vasculitis. Clinical and laboratory features of 82 patients seen in private practice. Arch Dermatol 1984; 120: 484-9.

6. MeHRegan D. Pathophysiology of urticarial vasculitis. Arch Dermatol 1998; 134: 88-9.

7. Fierro J, Cuchacovic M, Rodríguez M, Parra $M$, Sото M. Estudio clínico y anatomopatológico en 5 casos de vasculitis portadores de anticuerpos anticitoplasmáticos de neutrófilos con patrón citoplasmático. Rev Méd Chile 1995; 123: 841-8.

8. Savage C, Winearls C, Evans D. Microscopic polyarteritis: presentation, pathology and prognosis. Q J Med 1985; 56: 467-83.

9. BuRRows NP, Lochwood CM. Antineutrophil cytoplasmic antibodies and their relevance to the dermatologist. Review. Br J Dermatol 1995; 132: 173-81.

10. LANGFoRD C. Vasculitis. J Allergy Clin Immunol 2003; 111: 602-12.

11. Frances C, Thinuong L, Piette J, Saada V, Boisnic S, WeChSLER B ET AL. Wegener's granulomatosis. sostenerse en elementos clínicos más la suma de hallazgos histopatológicos cutáneos interpretados en el contexto del dinamismo del proceso inflamatorio vascular.

Dermatological manifestations in 75 cases with clinicopathologic correlation.

12. López de Maturana D, Amaro P, Balestrini C, Segovia L Manifestaciones clínicas en 5 casos de enfermedad de Behçet. Rev Méd Chile 2002; 130: 551-6.

13. Su WPD, Schroeter AL, Perry HO, Powell FC. Histopathologic and immunopathologic study of pyoderma gangrenosum. J Cutan Pathol 1986; 13: 323-30.

14. Kazmierowski J, Chusid M, Parrilo J, Fauci A, WolfF S. Dermatologic manifestations of the hypereosinophilic Syndrome. Arch Dermatol 1978; 114: 531-5.

15. Beylot J, Malou M, Doutre MS. Leukocytoclastic vasculitis and malignant hematologic diseases (12 cases). Rev Med Interne 1989; 10: 509-14.

16. JoNES RE. What is your criteria for identifying vasculitis of small vessels by conventional microscopy? Am J Dermatopathol 1985; 7: 181-7.

17. WinKelmanN RK, DitTo W. Cutaneous and visceral syndromes of necrotizing and allergic angeitis. A study of 38 cases. Medicine 1964; 43: 59-89.

18. Handel D, Roenigk $H$, Shainoff J, Deodhar $S$. Necrotizing vasculitis. Etiologic aspects of immunology and coagulopathy. Arch Dermatol 1975; 111: 847-52.

19. Cribier B, Couiliet D, Meyer P, Grosshaus E. The severity of histopathological changes of leukocitoclastic vasculitis is not predictive of extracutaneous involvement. Am J Dermatopathol 1999; 21: 532-6.

20. Sais G, Vidaler A, Jucgla A, Servitje O, Condom E, Peyri J. Prognostic factors in leukocitoclastic vasculitis. A clinicopathologic study of 160 patients. Arch Dermatol 1998; 134: 309-15. 